\title{
T-box transcription factor brachyury promotes tumor cell invasion and metastasis in non-small cell lung cancer via upregulation of matrix metalloproteinase 12
}

\author{
ZONGMIAO WAN $^{1 *}$, DONGJIE JIANG ${ }^{1 *}$, SU $_{\text {CHEN }}{ }^{1}$, JIAN JIAO ${ }^{1}$, LEI JI ${ }^{2}$, ABDUS SABOOR SHAH ${ }^{2}$, \\ HAIFENG WEI $^{1}$, XINGHAI YANG ${ }^{1}$, XIAOTAO LI $^{2}$, YING WANG ${ }^{3}$ and JIANRU XIAO ${ }^{1}$ \\ ${ }^{1}$ Department of Orthopedic Oncology, Changzheng Hospital, Second Military Medical University, Shanghai 200003; \\ ${ }^{2}$ Shanghai Key Laboratory of Regulatory Biology, Institute of Biomedical Sciences, East China Normal University, \\ Shanghai 200241; ${ }^{3}$ The Institute of Aging Research, School of Medicine, Hangzhou Normal University, \\ Hangzhou, Zhejiang 310036, P.R. China
}

Received November 10, 2015; Accepted December 17, 2015

DOI: $10.3892 /$ or.2016.4792

\begin{abstract}
T-box transcription factor brachyury and matrix metalloproteinases (MMPs) play important roles in nonsmall cell lung cancer (NSCLC) cell invasion and metastasis. However, the association between Brachyury and the MMP family has not yet been fully investigated. The present study aimed to assess the influence of Brachyury on the expression of $23 \mathrm{MMP}$ members and to further explore the mechanisms involved in the promotion of NSCLC cell invasion by Brachyury and MMPs in the H460 and H1299 stable cell lines. The protein expression levels and correlations between the brachyury transcription factor and the targeted MMPs were also validated in 52 NSCLC patient tissue samples. We observed that brachyury significantly upregulated MMP12 expression to promote NSCLC cell invasion. We also found a potential binding site for the brachyury transcription factor in the MMP12 promoter.
\end{abstract}

\section{Introduction}

Brachyury is the embryonic transcription factor of the T-box family (1), which has been reported as a prognostic factor in

Correspondence to: Dr Jianru Xiao, Department of Orthopedic Oncology, Changzheng Hospital, Second Military Medical University, 415 Fengyang Road, Shanghai 200003, P.R. China

E-mail: jianruxiao83@163.com

Dr Ying Wang, The Institute of Aging Research, School of Medicine, Hangzhou Normal University, 16 Xuelin Road, Hangzhou, Zhejiang 310036, P.R. China

E-mail: flashingdancer@163.com

${ }^{*}$ Contributed equally

Key words: brachyury, transcription factor, non-small cell lung cancer, matrix metalloproteinase 12 , metastasis various types of carcinomas such as gastric, colon, breast, prostate, esophageal, ovarian, and particularly in non-small cell lung cancer (NSCLC), which is one of the leading causes of cancer-related death worldwide (2-4). Brachyury protein expression has been reported in $41 \%$ of primary lung carcinomas, including $48 \%$ of adenocarcinomas and $25 \%$ of squamous cell carcinomas (5). Fernando et al (6) reported that overexpression of brachyury in human carcinoma cells induced changes characteristic of epithelial-mesenchymal transition (EMT), including upregulation of mesenchymal markers, downregulation of epithelial markers and an increase in cell migration and invasion. Haro et al (2) examined 104 primary lung carcinoma samples and found that expression of brachyury mRNA was significantly correlated with reduced 5-year disease-free and overall survival rates and was significantly correlated with vascular invasiveness, lymphatic permeation and lymph node metastasis. Xu et al (7) showed that expression of brachyury was significantly higher in 115 NSCLC tissue samples than that in adjacent normal lung tissues.

Matrix metalloproteinases (MMPs) are zinc-dependent endopeptidases and 23 members of the MMP family have been reported to date. Although MMPs exhibit a wide range of biological functions in cancer, their primary function is to degrade proteins in the extracellular matrix (ECM) and mediate cell-cell adhesion, which are implicated in EMT $(8,9)$. Previous studies have reported that MMP2 $(10,11)$, MMP7 (12,13), MMP9 (10,14), MMP10 (15), MMP12 (16,17), MMP14 (18,19), MMP19 (20) and MMP26 (21) are significantly overexpressed in NSCLC tissues and are correlated with poor patient prognosis.

MMPs and brachyury play an important role in lung cancer cell invasion and metastasis. However, the correlation between brachyury expression and MMP family genes has not been investigated in non-small cell lung carcinomas. In the present study, we aimed to explore the correlation between brachyury and MMP members, and also further explore the mechanism involved in the promotion of lung cancer metastasis by brachyury. 


\section{Materials and methods}

Tumor cell lines and reagents. H1299, H460 and HEK293 cells in the present study were purchased from the American Type Culture Collection (ATCC; Gaithersburg, MD, USA) and propagated in RPMI-1640 medium or Dulbecco's modified Eagle's medium (DMEM) (Gibco) supplemented with $10 \%$ fetal bovine serum (FBS) (Invitrogen) and 1\% penicillin and streptomycin in a cell incubator $\left(37^{\circ} \mathrm{C}, 5 \% \mathrm{CO}_{2}\right)$.

The antibodies for MMP12 (cat. \#ab52897; Abcam $^{\circledR}$ ), brachyury (cat. \#sc-17743 and cat. \#sc-20109), GAPDH (cat. \#sc-32233), Hsp90 (cat. \#sc-7949) (all from Santa Cruz Biotechnology, Inc., Santa Cruz, CA, USA) were purchased and used in the present study. HRP-goat anti-rabbit IgG and HRP-goat anti-mouse IgG were purchased from Jackson ImmunoResearch Laboratories. Transfection of DNA and RNAi was performed using Lipofectamine 2000 from Life Technologies (cat. \#11668019).

Plasmids and shRNAs. A lentivirus vector expressing human brachyury and lentivirus particles were obtained from Shanghai R\&S Biotechnology (Shanghai, China). In brief, human brachyury cDNA amplified from lung-derived cDNA samples was inserted into the Pac1/Nhe1 restriction enzyme sites within the multiple cloning site of the lentivirus vector pLenti6.3-IRES2-EGFP/V5 DEST, resulting in the vector pLenti-Brachyury-EGFP. Three siRNAs used to selectively silence the expression of brachyury with the given sequences (CCUCGAAUCCACAGUGATT, GCAAAUCCUCAUCCUC AGUTT and CUCCAACCUAUUCUGACAATT) were synthesized by Shanghai GenePharma Co., Ltd., while the sequence for the negative control was (UUCUCCGAACGUGUCACG UTT), as previously reported (22). The most efficient siRNA special for Brachyury in the H460 cells line was selected for the following shRNA construction (23), which targets ORF of Brachyury (+1021/+1041 bp). Plenti6.3-IRES-EGFP/V5 DEST was used to construct Bra-shRNA and con-shRNA according to shRNA construction protocol from Shanghai R\&S Biotechnology (Shanghai, China).

Transfection and culture of the stable cells. Transfection of the PGL3-Basic and pcDNA3.1-Flag-Brachyury plasmids was performed using Lipofectamine 2000 (cat.\#11668019) following the manufacturer's instructions. Basically, 5x105 H1299 cells were seeded in a $6-\mathrm{cm}$ culture dish overnight before transfection. Plasmid DNA $(2 \mu \mathrm{g})$ and $6 \mu$ l Lipofectamine 2000 reagent were gently mixed in $500 \mu 1$ RPMI-1640 medium without serum separately at room temperature for $5 \mathrm{~min}$. The diluted DNA was combined with the diluted Lipofectamine 2000 and incubated for $20 \mathrm{~min}$. The mixture was added into a culture dish drop by drop. Medium was replaced after $24 \mathrm{~h}$.

The constructed plenti 6.3-Brachyury/shRNA plasmids and packaging mix (pLP1-Gag-pol, pLP-VSVG and pLP2-Rev) were co-transfected into H293T cells using POLOdeliverer ${ }^{\mathrm{TM}} 3000$ transfection reagent (cat. \#CT001-1; Shanghai R\&S Biotechnology). The virus supernatant medium was collected after H293T cell infection for $48 \mathrm{~h}$. The mixture was centrifuged at $3,000 \mathrm{rpm} \times 15 \mathrm{~min}$ at $4^{\circ} \mathrm{C}$. Then, the liquid was filtered using a $0.45-\mu \mathrm{m}$ filter membrane. The acquired virus was stored at $-80^{\circ} \mathrm{C}$ until use. H1299-
pBra (brachyury-overexpressing) and H1299-pcon (negative control), H460-pshT (Brachyury-knockdown) and H460-pshN stable cells were infected, and were then selected after the processes of fluorescence activated cell sorting (GFP selection) and Blasticidin treatment (cat. \#60218ES50; InvivoGen).

Semi-quantitative PCR and real-time PCR. Reversetranscription PCR was performed using the SuperScript III One-Step RT-PCR system from Life Technologies (cat. \#1257418) following the protocol provided in the manual. Total RNA $(2 \mu \mathrm{g})$ was used for each reverse transcript reaction. The mRNA expression of 23 MMP members were semi-quantitatively measured via $2 \%$ agarose gel for H1299pCon, H1299-pBra, H460-pshN and H460-pshT cells. Each experiment was performed in duplicate and was repeated 3 times. The MMP members were subjected to real-time PCR using SYBR-Green Master Mix (Bio-Rad) and the Mx3005Pquantitative RT-PCR system (Stratagene) according to the semi-quantitative PCR results. Mean $\mathrm{Ct}$ values for the target genes were normalized to the mean $\mathrm{Ct}$ values for the endogenous control $18 \mathrm{~s}[-\Delta \mathrm{Ct}=\mathrm{Ct}(18 \mathrm{~s})-\mathrm{Ct}$ (target gene) $]$. The ratio of mRNA expression of the target gene vs. $18 \mathrm{~s}$ was defined as $2^{-\Delta \mathrm{Ct}}$.

Western blot analysis. Total protein extracts of each group were resolved by $11 \%$ SDS-PAGE and transferred onto polyvinylidene difluoride (PVDF) (Millipore) membranes. After blocking with $5 \%$ not-fat milk for $1 \mathrm{~h}$ at room temperature, the PVDF membranes were washed 3 times for 10 min with phosphate-buffered saline (PBS) followed by incubation with the primary antibodies [goat anti-human brachyury $(1: 8,000$; 3\% BSA dilution), rabbit anti-human MMP12 (1:250; 3\% no-fat milk dilution) and mouse anti-human HSP90 (1:4,000; $3 \%$ BSA dilution)] overnight at $4^{\circ} \mathrm{C}$. Following extensive washing, the membranes were incubated with secondary antibodies for $2 \mathrm{~h}$. After washing 3 times for $10 \mathrm{~min}$ with Trisbuffered saline/Tween-20 (TBST) at room temperature once more, the immunoreactivity was visualized using an ECL kit (cat. \#SK6668-100; Sangon Biotech) and the membranes were exposed to Kodak XAR-5 film (F5263; Sigma-Aldrich, USA).

Matrigel invasion assay. Blind well chambers (Neuro Probe Inc.) with $8-\mu \mathrm{m}$ pore polycarbonate filters coated with Matrigel (BD Biosciences) were used. RPMI-1640 medium supplemented with $10 \%$ FBS was added to the lower chambers; cells $\left(1 \times 10^{5}\right)$ were added in serum-free medium to the upper chambers. After a $24-\mathrm{h}$ incubation at $37^{\circ} \mathrm{C}$, the filters were fixed and stained with $0.1 \%$ solution of crystal violet. Cells on the bottom side of the filters were counted in five random x 100 objective microscopic fields. Experiments were conducted in triplicate for each cell line. Two MMP12 siRNAs were synthesized (Shanghai GenePharma, Co., Ltd.) as previously reported (24). The sequence for MMP12-siRNA1 was 5'-GCUGUUUUU AACCCACGUUTT-3'; and the sequence for MMP12-siRNA2 was 5'-CCGUGAGGAUGUUGACUACTT-3'. Negative control siRNA was also used.

Immunohistochemical staining. Immunohistochemical staining (IHC) was carried out following a previously published protocol (25). Specimens were deparaffinized with 
Table I. Demographic data of the cases used for immunohistochemistry.

\begin{tabular}{lcc}
\hline & $\begin{array}{c}\text { Primary } \\
\text { NSCLC }\end{array}$ & $\begin{array}{c}\text { Metastatic } \\
\text { NSCLC }\end{array}$ \\
\hline Mean age, in years & 61.9 & 60.8 \\
Minimum age & 46 & 41 \\
Maximum age & 82 & 73 \\
Gender, $\mathrm{n}$ & & \\
Male & 25 & 17 \\
Female & 5 & 5 \\
Histological type, $\mathrm{n}$ & & \\
Adenocarcinoma & 14 & 16 \\
Squamous carcinoma & 13 & 5 \\
Large cell carcinoma & 3 & 1 \\
\hline
\end{tabular}

Metastastic NSCLC from patients diagnosed with spine metastasis from NSCLC. NSCLC, non-small cell lung cancer.

xylene, subjected to antigen repair, and were blocked for 15 min with $5 \%$ goat serum. The slides were incubated with the anti-brachyury (1:400 dilution) and anti-MMP12 (1;200 dilution) antibodies at $4{ }^{\circ} \mathrm{C}$ overnight, followed by incubation with anti-rabbit second antibodies at room temperature for 30 min. Positive expression of IHC was reflected as brown staining estimated by averaging the numbers of positively stained cells under $\mathrm{x} 200$ high power vision fields. The demographic information of the NSCLC cases used for IHC is provided in Table I. The present study was approved by the Institutional Review Board of Shanghai Changzheng Hospital. Informed consent was obtained before tissue collection. Additionally, the Ethics Committee of Shanghai Changzheng Hospital approved the use of the obtained patient specimens for this study.

ChIP assay. Brachyury transcription factor cDNA was directionally cloned between XhoI and HindIII sites of the pCDNA vector which contained an $\mathrm{N}$-terminal flag tag. Sequencing analysis was performed to verify the construct. H1299 cells were transfected with constructed pcDNA3.1-flag-Brachyury, followed by chromatin immunoprecipitation assay (ChIP). Briefly, nuclear proteins of the transfected H1299 cells were cross-linked and sonicated to obtain DNA fragments of 200-1000 bp. The lysates were diluted in ChIP dilution buffer and then incubated with salmon sperm DNA/protein A agarose slurry (Invitrogen). The chromatin solutions were incubated with anti-flag tag (Sigma) and RNA pol2 (cat. \#sc-899; Santa Cruz Biotechnology) antibodies overnight at $4^{\circ} \mathrm{C}$ with rotation. Beads were pelleted by centrifugation. The IgG was used as a negative control. PCR amplification of the genomic fragments was performed with 10 specific primers flanking putative binding sites on the MMP12 promoter. The PCR products were separated by $2.0 \%$ agarose gel electrophoresis.

MMP12 luciferase reporter constructs and luciferase assay. The brachyury promoter sequence $(-1 /-1800 \mathrm{bp})$ was directionally cloned between the XhoI and HindIII sites of the pGL3-basic vector with the following sense primer: 5'-CCGCTCGAGTGTGGCTTTGGATAAGTTAAGTTCA-3' and antisense primer, 5'-CCCAAGCTTTGTAAACTTCT AAACGGATCAATTC-3'. Sequencing was performed to verify the construct. Three subcloning vectors of point mutation were performed using Site-Directed Mutagenesis kit (cat. \#D0206; Shanghai Beyotime, China) according to the manufacturer's protocol. Primers of pGL3-mmp12-MT1 (mutation at -959/-953) and pGL3-mmp12-MT2 (mutation at -923/-917) were: 5'-GAGGCAGCGAACTTATGGCTCGA TTTGAAGGGGTGTTGA-3' (forward) and 5'-TCAACA CCCCTTCAAATCGAGCCATAAGTTCGCTGCCTC-3' (reverse); 5'-TTGAGTAGAATCTATCCTCGTGCCGCAG CACCACTGCTT-3' (forward), and 5'-AAGCAGTGGTGC TGCGGCACGAGGATAGATTCTACTCAA-3' (reverse), respectively. PGL3-mmp12-MT1+2 (mutation at -959/-953 and -923/-917) was also constructed. These constructs were verified by sequencing.

After transfection and/or treatment, the cells were washed with PBS 3 times. The cells were then lysed in luciferase cell culture lysis buffer provided with the Dual-Luciferase Assay kit (Promega). After $-80^{\circ} \mathrm{C}$ cryopreservation for $1 \mathrm{~h}$ and rotation at room temperature for $20 \mathrm{~min}$, the whole cell lysates were collected into EP tubes and centrifuged at $4^{\circ} \mathrm{C}$ at $12,000 \mathrm{x} \mathrm{g}$ for $10 \mathrm{~min}$. The supernatant was collected in a fresh tube and $20 \mu 1$ of the supernatant was added to the luciferase assay substrate $(20 \mu \mathrm{l})$. Luminescence was measured as relative light units, twice for each lysate. Readings for the luciferase assay were carried out using LUMIstar OPTIMA (BMG Labtech). Each assay was repeated 3 times. Fold repression values were represented as mean of three experiments.

Statistical analysis. All of the measurements were collected in triplicate for each independent operation. The data were statistically analyzed using the Student's t-test. Statistical analysis was carried out using SPSS 16.0 statistical software (SPSS, Inc., Chicago, IL, USA). Statistical significance was set as a p-value $<0.05$.

\section{Results}

MMP12 was significantly correlated with brachyury expression in the NSCLC cell lines. T-box transcription factor brachyury has been recently reported as a poor prognostic factor in various types of carcinomas and is mainly characterized as a driver of EMT $(5,6)$. MMP family members play an important role in tumor cell metastasis promoted by the brachyury transcription factor in NSCLC. However the correlation between brachyury and MMPs remains unclear. We constructed and selected two stable cell lines (H1299pBra and H460-pshT) and two negative control stable cell lines (H1299-pCon and H460-pshN). mRNA expression of brachyury in the H1299-pBra cells was significantly increased by 64.8 -fold $(\mathrm{p}<0.001)$, compared with that in the H1299pCon cells (Fig. 1A). mRNA expression of brachyury in the H460-pshT cells was significantly reduced by $73 \%(\mathrm{p}<0.001)$, compared with that in the H1299-pCon cells (Fig. 1B). Protein expression of brachyury in the H1299-pBra and H460-pshT cell lines also showed consistent results (Fig. 2A and B). 

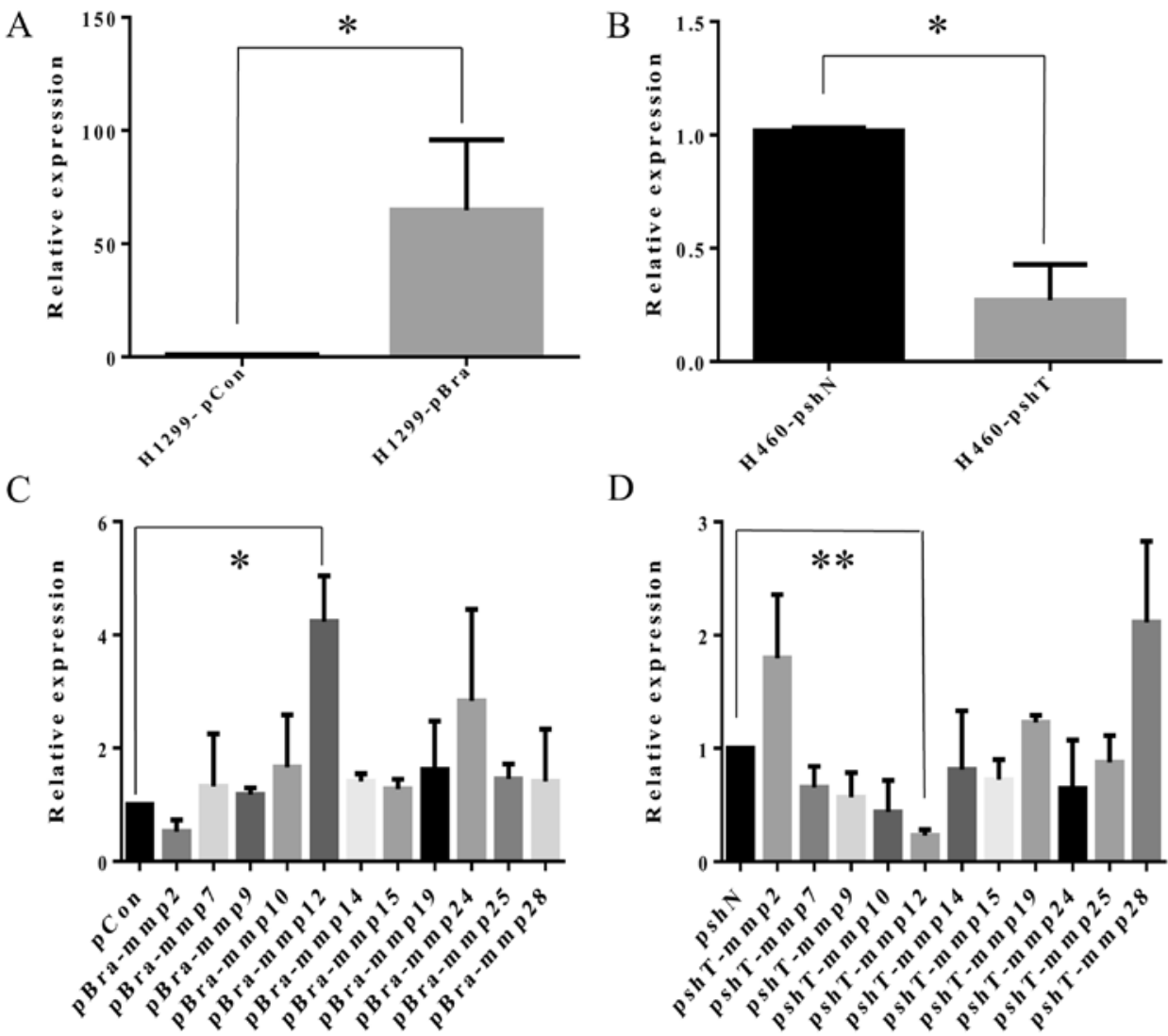

D

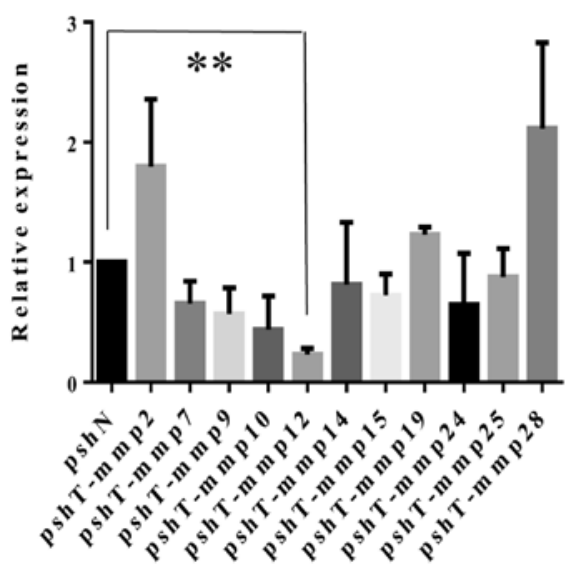

Figure 1. Expression of brachyury and 11 MMP members as determined by real-time PCR analysis in H1299 and H460 cell lines. (A) Relative expression of brachyury mRNA in H1299-pBra (brachyury stable overexpressing cellsn) compared with H1299-pCon cells (negative control). (B) Relative expression of brachyury mRNA in H460-pshT (brachyury stable knockdown cells by shRNA) compared with H460-pshN cells (shRNA negative control). (C) Relative expression levels of 11 MMP members as determined by RT-qPCR in the H1299-pBra cells compared with the H1299-pCon cells. (D) Relative expression levels of 11 MMP members as determined by RT-qPCR in the H460-pshT cells compared with the H460-pshN cells. ${ }^{*}$ p $<0.05,{ }^{* * *} \mathrm{p}<0.01$.

After extraction of total RNA and reverse transcription of cDNA from the 4 NSCLC stable cell lines (H1299-pBra, H1299-pCon, H460-pshT and H460-pshN) we investigated the mRNA expression levels of 23 MMP members by semiquantitative PCR. Furthermore, we selected 11 MMP members (MMP2, MMP7, MMP9, MMP10, MMP12, MMP14, MMP15, MMP19, MMP24, MMP25 and MMP28) for quantitative real-time PCR according to the agarose gel results (data not shown), and reported MMP members with increased expression in NSCLC tissues as reported in previous literature (10-20). mRNA expression levels of MMPs in the H1299-pBra cells were as follows. MMP2 exhibited a $47 \%$ decrease $(p=0.07)$, MMP7 exhibited a $32 \%$ increase ( $\mathrm{p}=0.61)$, MMP9 exhibited a $18 \%$ increase $(\mathrm{p}=0.11)$, MMP10 exhibited a $66 \%$ increase ( $\mathrm{p}=0.34)$, MMP12 exhibited a 323\% increase ( $\mathrm{p}=0.02)$, MMP14 exhibited a $41 \%$ increase $(\mathrm{p}=0.88)$, MMP15 exhibited a $28 \%$ increase $(\mathrm{p}=0.94)$, MMP19 exhibited a $62 \%$ increase $(\mathrm{p}=0.59)$, MMP24 exhibited a 183\% increase ( $\mathrm{p}=0.40)$, MMP25 exhibited a $46 \%$ increase $(\mathrm{p}=0.86)$, MMP2 8 exhibited a $8 \%$ increase $(\mathrm{p}=0.60)$, compared with the relative levels in the negative control H1299-pCon cells (Fig. 1C). mRNA expression levels of MMPs in the H460-pBra cells were as follows. MMP2 exhibited an $80 \%$ increase $(\mathrm{p}=0.13)$, MMP7 exhibited a $34 \%$ decrease $(\mathrm{p}=0.09)$, MMP9 exhibited a 43\% decrease $(\mathrm{p}=0.08)$, MMP10 exhibited a 56\% decrease ( $\mathrm{p}=0.07)$, MMP12 exhibited a $77 \%$ decrease $(\mathrm{p}<0.001)$, MMP14 exhibited a $19 \%$ decrease
A

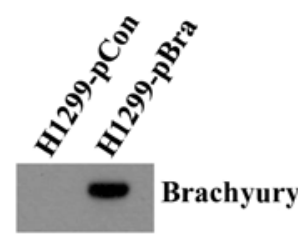

B

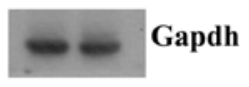

C

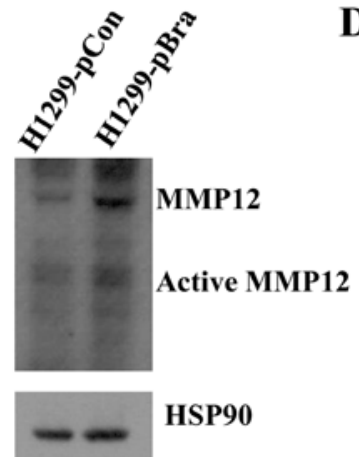

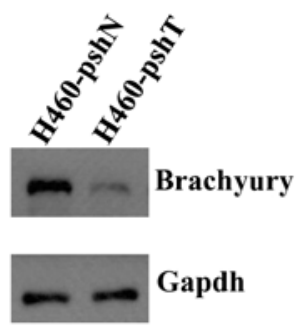

D
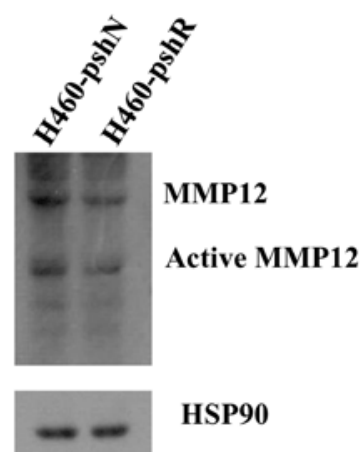

Figure 2. Expression of brachyury and MMP12 as determined by western blot experiments. (A) Relative expression levels of Brachyury protein in H1299-pBra and H1299-pCon stable cells. (B) Relative expression levels of Brachyury protein in H460-pshT and H460-pshN cells. (C) Western blot analysis of MMP12 protein expression in H1299-pBra and H1299-pCon cells. (D) Western blot analysis of MMP12 protein expression in H460-pshT and H460-pshN cells. 
A

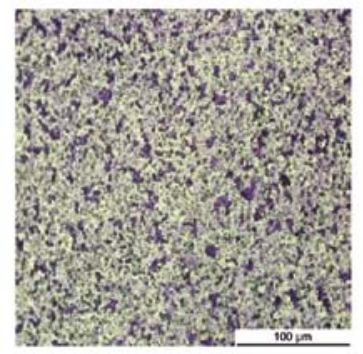

pCon-NC

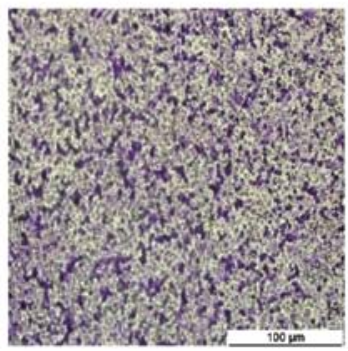

pBra-NC

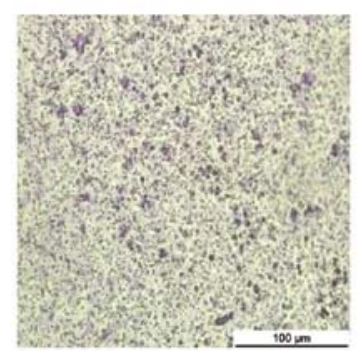

pCon-siRNA1

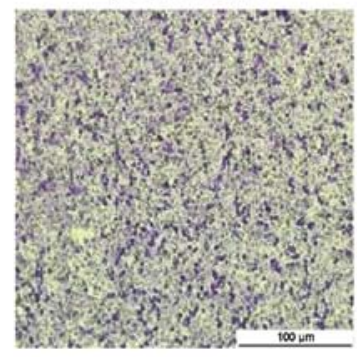

pBra-siRNA1

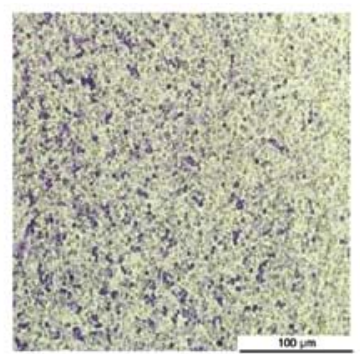

pCon-siRNA2

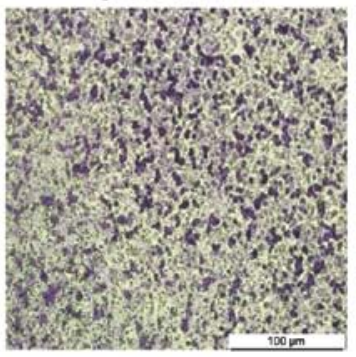

pBra-siRNA2
B

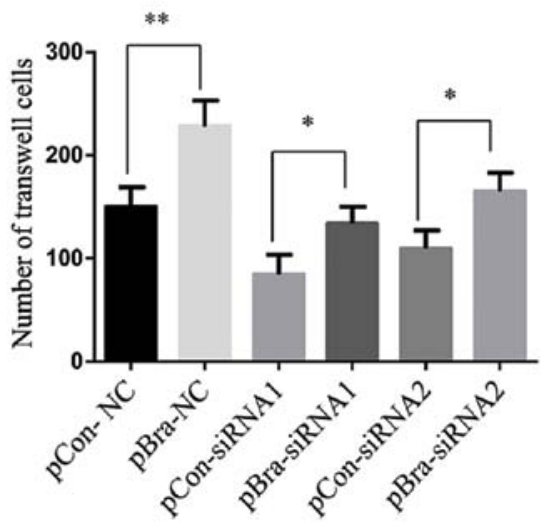

C

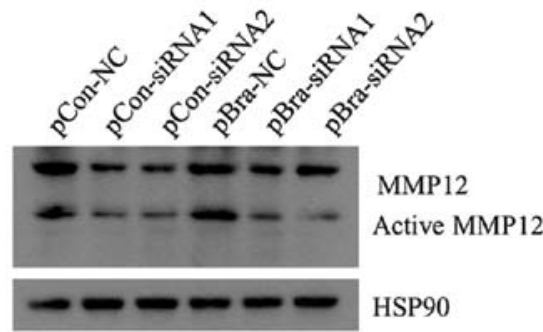

Figure 3. Brachyury upregulates MMP12 expression to promote NSCLC cell invasion. (A) Representative results of the Matrigel invasion assay of H1299pCon (brachyury negative control) and H1299-pBra (brachyury-overexpressing) stable cells, which were treated with MMP12-siRNA-NC, MMP12-siRNA1 and MMP12-siRNA2. Scale bars, $100 \mu \mathrm{m}$. (B) Statistical analysis of Matrigel invasive cells between H1299-pCon-NC (H1299-pCon stable cells transfected with MMP12-siRNA-NC) and H1299-pBra-NC (H1299-pBra stable cells transfected with MMP12-siRNA-NC), between H1299-pCon-siRNA1 (H1299pCon stable cells transfected with MMP12-siRNA1) and H1299-pBra-siRNA1 (H1299-pBra stable cells transfected with MMP12-siRNA1), and between H1299-pCon-siRNA2 (H1299-pCon stable cells transfected with MMP12-siRNA2) and H1299-pBra-siRNA2 (H1299-pBra stable cells transfected with MMP12-siRNA2). " $\mathrm{p}<0.05$; ${ }^{* *} \mathrm{p}<0.01$. (C) Western blot analysis of MMP12 was performed in the H1299-pCon-NC, H1299-pCon-siRNA1, H1299-pConsiRNA2, H1299-pBra-NC, H1299-pBra-siRNA1 and H1299-pBra-siRNA2.

( $\mathrm{p}=0.68)$, MMP15 exhibited a 28\% decrease $(\mathrm{p}=0.77)$, MMP19 exhibited a $23 \%$ increase $(\mathrm{p}=0.04)$, MMP24 exhibited a $35 \%$ decrease $(\mathrm{p}=0.29)$, MMP25 exhibited a $12 \%$ decrease $(\mathrm{p}=0.08)$, MMP28 exhibited a $110 \%$ increase $(\mathrm{p}=0.06)$, compared with the levels in the negative control H1299-pCon cells (Fig. 1D). We concluded that the expression of MMP12 was the most significantly correlated with the expression of brachyury. Consistent with the mRNA expression of MMP12 and brachyury, we observed that the protein level of MMP12 (54 kDa) and the active protein level of MMP12 (45 kDa) were increased in the H1299-pBra cells as compared to the H1299-pCon cells. The protein level of MMP12 and the active protein level of MMP12 were decreased in the H460-pshT cells as compared to the H460-pshN cells (Fig. 2C and D).

Brachyury promotes NSCLC cell invasion via MMP12 expression. Next, we investigated the role of MMP12 in the promotion of NSCLC cell invasion by brachyury. We synthesized two MMP12 siRNAs for transfection into the H1299-pBra and H1299-pCon stable cells.

As shown in Fig. 3C, the MMP12 protein levels were reduced in the H1299-pCon and H1299-pBra cell lines following transfection of the two synthesized siRNAs targeting MMP12, compared to the levels in the cells transfected with a control siRNA. Importantly, the H1299-pBra cells displayed high protein expression of MMP12 compared with the level in the H1299-pCon cells, when we performed the same MMP12siRNA treatment suggesting that brachyury promotes the expression of MMP12.

Consistent with the protein expression between brachyury and MMP12, the Matrigel invasion assay revealed higher invasive ability in the H1299-pBra cells than that in the H1299-pCon cells following treatment with MMP12-NC or MMP12-siRNA1 and MMP12-siRNA2 (Fig. 3). Statistical analysis between 
A

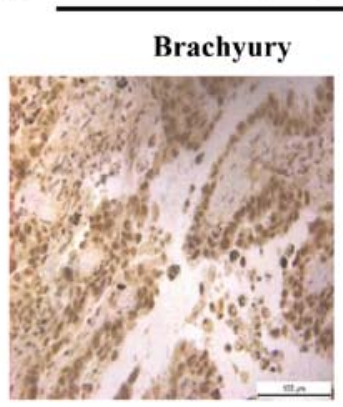

High expression

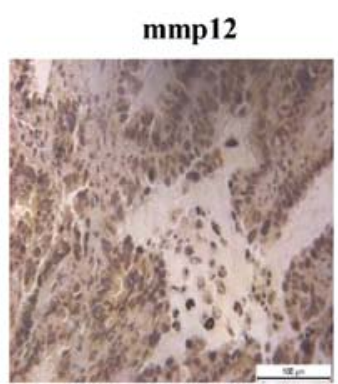

B

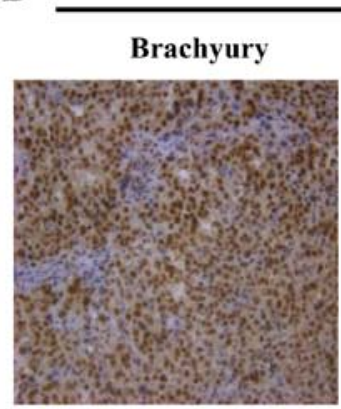

High expression

C

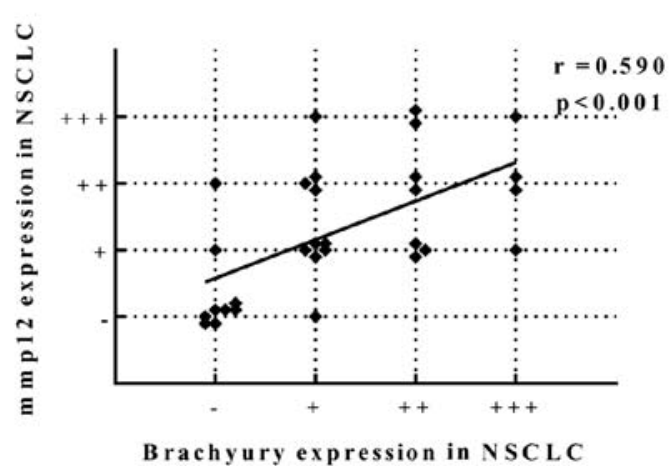

Low expression

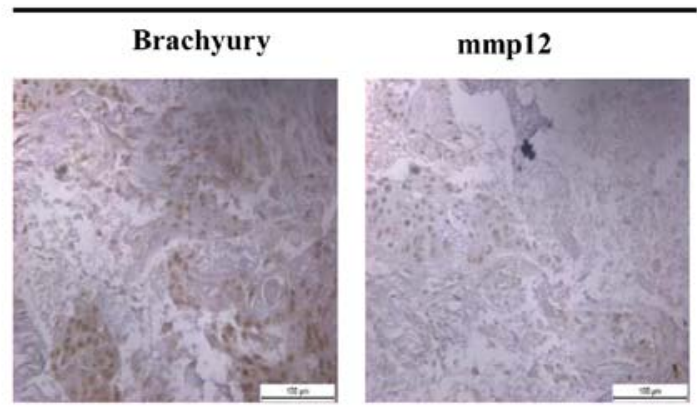

Low expression

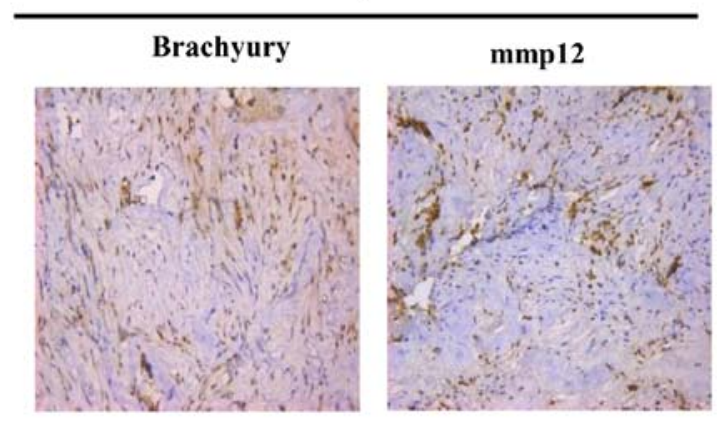

D

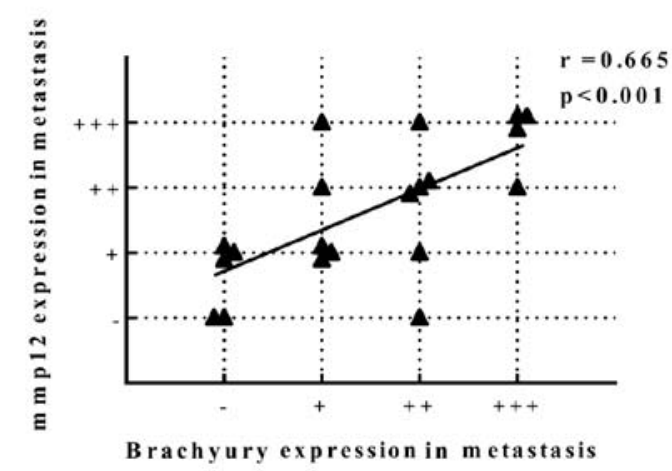

Figure 4. IHC staining of brachyury and MMP12. IHC staining was performed using adjacent sections of the same sample to detect the correlation between brachyury and MMP12. Immunohistochemical results were evaluated independently by two pathologists independently in a blinded randomized manner. Scatter plot for brachyury and MMP12 correlation in the same sets of tumors. The scores were put into the plot using the Bland-Altman plot standard method. In addition, non-parametric Spearman correlation tests were used. Significance was defined as p<0.05. Magnification, x200. Scale bars, $100 \mu$ m. (A) Strong staining of Brachyury in one primary NSCLC case which also showed strong staining of MMP12 (right panel); reduced staining of brachyury in one primary NSCLC case, which also showed medium staining of MMP12 (left panel). (B) Strong staining of brachyury in one NSCLC metastasis case, which also showed strong staining of MMP12 (right panel); reduced staining of brachyury in one NSCLC metastasis case, which also showed medium staining of MMP12 (left panel). (C) Correlation between brachyury and MMP12 protein expression in 30 primary NSCLC cases. (D) Correlation between brachyury and MMP12 protein expression in 22 NSCLC metastasis cases.

the H1299-pCon-NC and H1299-pBra-NC cells; between H1299-pCon-siRNA1 and H1299-pBra-siRNA1 cells; between H1299-pCon-siRNA2 and H1299-pBra-siRNA2 cells, showed a significant reduction in invasive ability $(\mathrm{p}<0.05)($ Fig. 3B).

Expression of MMP12 is correlated with the brachyury level in human primary lung cancer samples and metastasis samples from lung cancer disease. Expression levels of brachyury and MMP12 protein were assessed by IHC in a series of 30 primary NSCLC carcinomas and 22 NSCLC metastatic tissues. Fig. 4A and B shows representative results of the intensity scores observed for brachyury and MMP12 expression, suggesting a positive correlation between brachyury and MMP12 in the primary NSCLC carcinomas and metastatic tissues. Brachyury expression exhibited a high level in $4 / 30$ cases, a medium level in 7/30 cases, a low level in 10/30 cases and a negative level in 9/30 cases in the primary NSCLC carcinoma tissues. MMP12 expression exhibited a high level in 4/30 cases, a medium level in $8 / 30$ cases, a low level in 10/30 cases and a negative level in $8 / 30$ cases. Furthermore, brachyury expression exhibited a high level in 4/22 cases, a medium level in 6/22 cases, a low level in 5/22 cases and a negative level in 5/22 cases in the lung cancer metastatic tissues. MMP12 expression exhibited a high level in 5/22 cases, a medium level in $5 / 22$ cases, a low level in $7 / 22$ cases and a negative level in 3/22 cases. We analyzed the correlation between brachyury and MMP12 in the same sets of tumors by scatter and agreement plots. These plots indicated that these two proteins were highly correlated in the same sets 


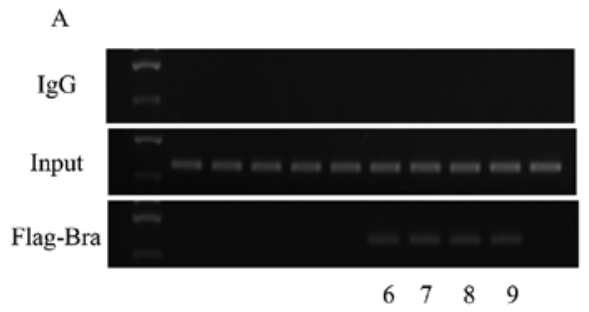

$\mathrm{C}$

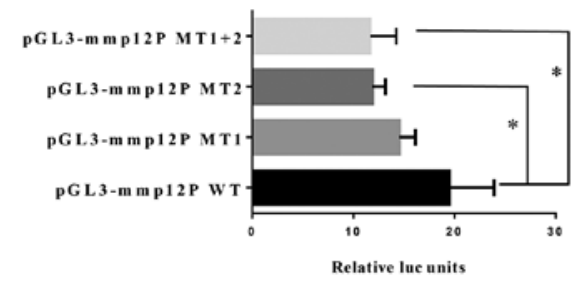

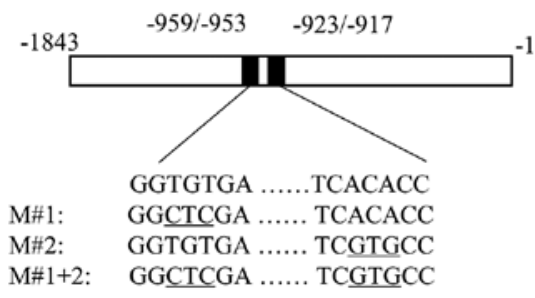

D

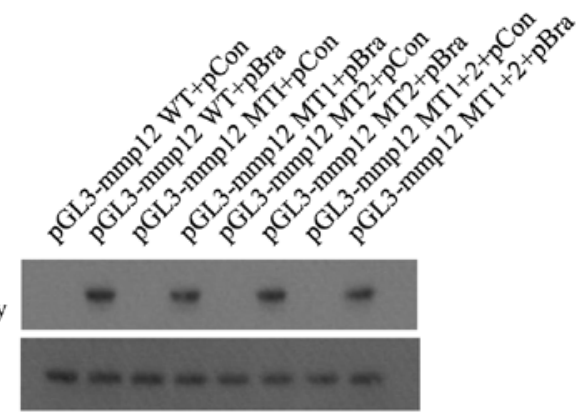

Figure 5. Transcriptional regulation of MMP12 by brachyury. (A) Chromatin immunoprecipitation was carried out using nuclear extracts from the H1299brachyury cells (transfected with pcDNA3.1-Flag-Brachyury plasmid) and an antibody against Flag, followed by RT-PCR analysis. Primers were designed for the 10 segments of the 5' MMP12 promoter sequence (-1/-3,087 bp). Negative control was performed using IgG. The pull-down DNA fragments is corresponding to the 6-9 parts of promoter sequence (-1443 bp to -266). (B) Schematic diagrams of PGL3 reported constructs, which contained mutated bases of the MMP12 promoter (-1/-1,843 bases). H1299 cells were pretreated with pcDNA3.1-Brachyury or pcDNA3.1-NC (Brachyury negative control), and co-transfected with pGL3-basic with mmp12P-MT1 (-959/-953 base mutation) or mmp12P-MT2 (-923/-917 base mutation) or mmp12P-MT1+2 (-959/-953 and -923/-917 base mutations). (C) Statistical analysis of pGL3-basic-mmp12P-WT (co-transfected with pcDNA3.1-Brachyury plasmid and pGL3-basic-mmp12P), pGL3-basicmmp12P MT1, pGL3-basic-mmp12P MT2 and pGL3-basic-mmp12P MT1+2. "p<0.05. (D) Western blot analysis of brachyury and GAPDH in the transfected H1299 cells, which were used for the mmp12 promoter luciferase reporter assay.

of tumor tissues. Pearson's correlation coefficient was used as a measure of correlation between brachyury and MMP12, which was 0.59 for primary lung cancer tissues (Fig. 4C) and 0.665 for lung cancer metastatic tissues (Fig. 4D).

Brachyury potentially regulates MMP12 in NSCLC cells. Since the mRNA and protein expression levels of MMP12 were correlated with brachyury, we sought to explore whether brachyury regulates MMP12 in the invasion and metastasis of NSCLC cells. We designed a CHIP-PCR for the MMP12 promoter in the $\mathrm{H} 1299$ cells transfected with the pcDNA3.1-Flag-Brachyury vector. The 5' MMP12 promoter sequence ( -3049 bp to $-1 \mathrm{bp}$ ) was divided into 10 parts, and 10 pairs of primers were designed to amplify each part of the promoter. The anti-Flag Tag antibody specifically pulled down DNA fragments corresponding to the -1443 bp to -266 bp region (Fig. 5A). This effect was not observed when an irrelevant control IgG was used.

Brachyury and other T-box transcription family members preferentially bind to the consensus elements AATTTCACAC CTAGGTGTGAAATT (6). Bioinformatic analysis revealed two putative half-site 5'-GGTGTGA-3' (-959/-953 bp) and 5'-TCACACC-3' (-923/-917 bp) within the region (Fig. 5B). Then, we performed 4 cloning plasmids including pGL3MMP12-WT (-1/-1,843 bp) and other three point mutation pGL3-basic plasmids (pGL3-MMP12-MT1, pGL3MMP12-MT2 and pGL3-MMP12-MT1+2) (Fig. 5B). MMP12 promoter luciferase reporter assay showed that the point mutations at each or both potential half-site of the consensus elements reduced the luciferase units, and the second position (-923/-917 bp) presented a more marked and significant reduction by $39.1 \%(\mathrm{p}<0.05)$ (Fig. $5 \mathrm{C})$. These samples of the luciferase reporter assay were subjected to western blot assay using GAPDH and brachyury antibodies (Fig. 5D).

\section{Discussion}

The product of the brachyury gene is a member of the T-box family of transcription factors, which is always highly present in various tumors yet is rarely observed in adult normal tissues $(26,27)$. The brachyury transcription factor can promote tumor cell invasion and distant metastasis in various types of cancer such as colorectal cancer, breast carcinoma, prostate cancer and NSCLC, and is also an independent prognostic factor in the clinic (4,28-30). In the present study, we found overexpression of brachyury and MMP12 in primary NSCLC and lung cancer metastatic tissues. Furthermore, brachyury promoted lung cancer cell migration and invasion mainly through upregulation of MMP12 in vitro. Moreover we observed a positive correlation between brachyury and MMP12 by investigating RNA and protein expression levels in H1299-pBra and H460-pshT stable cells, and in primary NSCLC and lung cancer metastatic patient tissues.

In accordance with our study, Roselli et al (5) reported that the expression of brachyury was significantly increased in human NSCLC samples compared with the adult normal tissues by PCR and IHC techniques. Haro et al (2) also found that brachyury mRNA expression in primary lung carcinoma tissues was significantly increased and was a predictor of poor prognosis. Hofmann et al (31) demonstrated that MMP12 expression was significantly increased in tumors vs. corresponding lung tissues and MMP12 expression was significantly correlated with local recurrence and metastatic disease by examination of the expression of 22 MMPs in 89 
surgically treated NSCLC patients. Kettunen et al (16) and Cho et al (17) also demonstrated that MMP12 was significantly overexpressed in NSCLC patient tissues using c-DNA microarray technique.

However, previous studies have not investigated the expression of brachyury and MMP12 in lung cancer metastasis tissues. In the present study, we also observed a significantly positive correlation between brachyury and MMP12. The patients were diagnosed with spine metastasis from NSCLC. Although it has been debated for decades how cancer cells acquire metastatic capability, distant metastatic cells not only have similar gene expression profiles, but also possess various discordant malignant features, compared with the primary carcinoma $(32,33)$. In line with our data, we observed a positive correlation of brachyury and MMP12 between NSCLC metastasis and the normal control lung tissues was more remarkable than between primary NSCLC tissues and the normal ones.

In vitro we found that the transcription factor brachyury had a significant effect on MMP12 expression and the promotion of migration and invasion of tumor cells by brachyury was associated with MMP12 in two adenocacinoma cell lines H460 and H1299. Consistent with our findings, Fernando et al (6) also found that brachyury overepression significantly promoted the invasion in tumor cell lines such as lung cancer cell line H460. Sarkar et al (34) reported that brachyury was expressed in a subpopulation of colorectal cancer cells that resemble invasive front mesenchymal-like cells with characteristics of cancer stem cells, allowing the cells to respond to signals prompting invasion or metastasis. Kobayashi et al (29) showed that knockdown of the brachyury transcription factor increased sensitivity of adenoid cystic carcinoma cells to chemotherapy and radiation in vitro. However, these previous studies did not deeply investigate the correlation between brachyury as an EMT driver and MMPs which play a crucial role in the process of tumor metastasis including the process of EMT and degradation of ECM. In our experiments, we found a distinct significant correlation between brachyury and MMP12 via PCR experiments, furthermore verifying the association in vivo. Different with our results, Fernando et al (6) observed that the $\mathrm{H} 460$ cell line with inhibition of brachyury expression concomitantly had reduced expression of MMP2 and MMP24. We also observed that MMP24 expression was increased by $183 \%$ concomitant following brachyury overexpression and was reduced by $35 \%$ concomitant with inhibiton of brachyury expression.

In the present study, a high level of MMP12 expression was correlated with increased expression of brachyury in NSCLC patients, suggesting that MMP12 is at least partially responsible for the brachyury transcription factor-mediated metastasis of NSCLC. CHIP and promoter luciferase assays, disclosed that the segment sequence -216 to $-1,083$ bp relative to the transcription start site of MMP12 with positive results of CHIP-PCR and luciferase units was also significantly increased by 13.6-fold when pGL3-basic vector was inserted the same segment sequence of MMP12 promoter. The sequence of 'AATTCACACCTAGGTGTGAAATT' is the conservative element preferentially bound by brachyury and other T-box transcription family (6). Bioinformatic analysis showed two half sites (GGTGTGA and TCACACC) concentrative to -862 to $-820 \mathrm{bp}$ relative to the transcription start site of
MMP12, which possibly were the binding sites by brachyury transcription factor complex. Point mutation and luciferase reporter assay showed point mutation at the MMP12 promoter sequence (-923/-917 bp) can significantly cause luciferase units with $39.1 \%$ reduction.

Recent studies have reported that brachyury is a driver of EMT, which allows the conversion of epithelial polarized and stationary cells into highly motile and invasive mesenchymal cells $(5,6,35)$. Fernando et al (6) reported that brachyury is able to bind to the E-cadherin promoter promoting EMT, an effect partially mediated by Slug. The present study contributes to further clarify the role and pathological mechanism of brachyury transcription factor in NSCLC migration and metastasis.

In conclusion, we showed for the first time that brachyury upregulated MMP12 in NSCLC tumors promoting cancer cell migration and invasion. Our data thus suggest brachyury as a potential therapeutic target for NSCLC. However, the detailed regulatory mechanisms in regards to MMP12 still require further investigation.

\section{Acknowledgements}

The present study was supported by the National Nature Science Foundation of China (81272943 and 81102036), and the Science and Technology Commission of Shanghai Municipality (12JC1411300). .

\section{References}

1. Kispert A, Koschorz B and Herrmann BG: The T protein encoded by Brachyury is a tissue-specific transcription factor. EMBO J 14: 4763-4772, 1995.

2. Haro A, Yano T, Kohno M, Yoshida T, Koga T, Okamoto T, Takenoyama M and Maehara Y: Expression of Brachyury gene is a significant prognostic factor for primary lung carcinoma. Ann Surg Oncol 20 (Suppl 3): S509-S516, 2013.

3. Imajyo I, Sugiura T, Kobayashi Y, Shimoda M, Ishii K, Akimoto N, Yoshihama N, Kobayashi I and Mori Y: T-box transcription factor Brachyury expression is correlated with epithelial-mesenchymal transition and lymph node metastasis in oral squamous cell carcinoma. Int J Oncol 41: 1985-1995, 2012.

4. Kilic N, Feldhaus S, Kilic E, Tennstedt P, Wicklein D, Wasielewski R, Viebahn C, Kreipe H and Schumacher U: Brachyury expression predicts poor prognosis at early stages of colorectal cancer. Eur J Cancer 47: 1080-1085, 2011.

5. Roselli M, Fernando RI, Guadagni F, Spila A, Alessandroni J, Palmirotta R, Costarelli L, Litzinger M, Hamilton D, Huang B, et al: Brachyury, a driver of the epithelial-mesenchymal transition, is overexpressed in human lung tumors: An opportunity for novel interventions against lung cancer. Clin Cancer Res 18: 3868-3879, 2012.

6. Fernando RI, Litzinger M, Trono P, Hamilton DH, Schlom J and Palena C: The T-box transcription factor Brachyury promotes epithelial-mesenchymal transition in human tumor cells. J Clin Invest 120: 533-544, 2010.

7. Xu K, Liu B and Liu Y: Impact of Brachyury on epithelialmesenchymal transitions and chemosensitivity in non-small cell lung cancer. Mol Med Rep 12: 995-1001, 2015.

8. Kleiner DE and Stetler-Stevenson WG: Matrix metalloproteinases and metastasis. Cancer Chemother Pharmacol 43 (Suppl): S42-S51, 1999.

9. Sternlicht MD and Werb Z: How matrix metalloproteinases regulate cell behavior. Annu Rev Cell Dev Biol 17: 463-516, 2001.

10. Wang R, Ke ZF, Wang F, Zhang WH, Wang YF, Li SH and Wang LT: GOLPH3 overexpression is closely correlated with poor prognosis in human non-small cell lung cancer and mediates its metastasis through upregulating MMP-2 and MMP-9. Cell Physiol Biochem 35: 969-982, 2015. 
11. Ali-Labib R, Louka ML, Galal IH and Tarek M: Evaluation of matrix metalloproteinase-2 in lung cancer. Proteomics Clin Appl 8: 251-257, 2014.

12. Zhang J, Luo J, Ni J, Tang L, Zhang HP, Zhang L, Xu JF and Zheng D: MMP-7 is upregulated by COX-2 and promotes proliferation and invasion of lung adenocarcinoma cells. Eur J Histochem 58: 2262, 2014.

13. Liang Y, Guo S and Zhou Q: Prognostic value of matrix metalloproteinase-7 expression in patients with non-small cell lung cancer. Tumour Biol 35: 3717-3724, 2014.

14. Ruiz-Morales JM, Dorantes-Heredia R, Arrieta O, ChavezTapia NC and Motola-Kuba D: Neutrophil gelatinase-associated lipocalin (NGAL) and matrix metalloproteinase-9 (MMP-9) prognostic value in lung adenocarcinoma. Tumour Biol 36: 3601-3610, 2015

15. Gill JH, Kirwan IG, Seargent JM, Martin SW, Tijani S, Anikin VA, Mearns AJ, Bibby MC, Anthoney A and Loadman PM: MMP-10 is overexpressed, proteolytically active, and a potential target for therapeutic intervention in human lung carcinomas. Neoplasia 6 : 777-785, 2004

16. Kettunen E, Anttila S, Seppänen JK, Karjalainen A, Edgren H, Lindström I, Salovaara R, Nissén AM, Salo J, Mattson K, et al: Differentially expressed genes in nonsmall cell lung cancer: Expression profiling of cancer-related genes in squamous cell lung cancer. Cancer Genet Cytogenet 149: 98-106, 2004.

17. Cho NH, Hong KP, Hong SH, Kang S, Chung KY and Cho SH: MMP expression profiling in recurred stage IB lung cancer. Oncogene 23: 845-851, 2004.

18. Wang YZ, Wu KP, Wu AB, Yang ZC, Li JM, Mo YL, Xu M, Wu B and Yang ZX: MMP-14 overexpression correlates with poor prognosis in non-small cell lung cancer. Tumour Biol 35: 9815-9821, 2014.

19. Zhou H, Wu A, Fu W, Lv Z and Zhang Z: Significance of semaphorin-3A and MMP-14 protein expression in non-small cell lung cancer. Oncol Lett 7: 1395-1400, 2014.

20. Yu G, Herazo-Maya JD, Nukui T, Romkes M, Parwani A, Juan-Guardela BM, Robertson J, Gauldie J, Siegfried JM, Kaminski N, et al: Matrix metalloproteinase-19 promotes metastatic behavior in vitro and is associated with increased mortality in non-small cell lung cancer. Am J Respir Crit Care Med 190: 780-790, 2014

21. Zhang Y, Zhao H, Wang Y, Lin Y, Tan Y, Fang X and Zheng L: Non-small cell lung cancer invasion and metastasis promoted by MMP-26. Mol Med Rep 4: 1201-1209, 2011.

22. Li J, Zhu L, Qu X, Li J, Lin R, Liao L, Wang J, Wang S, Xu Q and Zhao RC: Stepwise differentiation of human adipose-derived mesenchymal stem cells toward definitive endoderm and pancreatic progenitor cells by mimicking pancreatic development in vivo. Stem Cells Dev 22: 1576-1587, 2013.

23. Wang HZ, He YX, Yang CJ, Zhou W and Zou CG: Hepcidin is regulated during blood-stage malaria and plays a protective role in malaria infection. J Immunol 187: 6410-6416, 2011.
24. Sarkar S, Nuttall RK, Liu S, Edwards DR and Yong VW: Tenascin-C stimulates glioma cell invasion through matrix metalloproteinase-12. Cancer Res 66: 11771-11780, 2006.

25. Edwards YH, Putt W, Lekoape KM, Stott D, Fox M, Hopkinson DA and Sowden J: The human homolog $T$ of the mouse $T$ (Brachyury) gene; Gene structure, cDNA sequence, and assignment to chromosome 6q27. Genome Res 6: 226-233, 1996.

26. Ali A, Wang Z, Fu J, Ji L, Liu J, Li L, Wang H, Chen J, Caulin C, Myers JN, Zhang P, Xiao J, Zhang B and Li X: Differential regulation of the REG $\gamma$-proteasome pathway by $\mathrm{p} 53 / \mathrm{TGF}-\beta$ signalling and mutant p53 in cancer cells. Nat Commun 4: 2667, 2013.

27. Krukovskaia LL, Polev DE, Nosova Iu K, Baranova AV, Koliubaeva SN and Kozlov AP: Investigation of transcription factor Brachyury (T) expression in human normal and tumor tissues. Vopr Onkol 54: 739-743, 2008 (In Russian).

28. Palena C, Fernando RI, Hamilton DH, Huang B and Schlom J: Brachyury, a driver of tumor invasiveness and resistance to multiple therapies, is a novel immunotherapy target. J Immunother Cancer 1 (Suppl 1): P230, 2013.

29. Kobayashi Y, Sugiura T, Imajyo I, Shimoda M, Ishii K, Akimoto N, Yoshihama N and Mori Y: Knockdown of the T-box transcription factor Brachyury increases sensitivity of adenoid cystic carcinoma cells to chemotherapy and radiation in vitro: Implications for a new therapeutic principle. Int J Oncol 44: 1107-1117, 2014.

30. Pinto F, Pértega-Gomes N, Pereira MS, Vizcaíno JR, Monteiro P, Henrique RM, Baltazar F, Andrade RP and Reis RM: T-box transcription factor brachyury is associated with prostate cancer progression and aggressiveness. Clin Cancer Res 20: 4949-4961, 2014.

31. Hofmann HS, Hansen G, Richter G, Taege C, Simm A, Silber RE and Burdach S: Matrix metalloproteinase-12 expression correlates with local recurrence and metastatic disease in non-small cell lung cancer patients. Clin Cancer Res 11: 1086-1092, 2005.

32. Weigelt B, Glas AM, Wessels LF, Witteveen AT, Peterse JL and van't Veer LJ: Gene expression profiles of primary breast tumors maintained in distant metastases. Proc Natl Acad Sci USA 100: 15901-15905, 2003.

33. Gomez-Roca C, Raynaud CM, Penault-Llorca F, Mercier O, Commo F, Morat L, Sabatier L, Dartevelle P, Taranchon E, Besse B, et al: Differential expression of biomarkers in primary non-small cell lung cancer and metastatic sites. J Thorac Oncol 4: 1212-1220, 2009

34. Sarkar D, Shields B, Davies ML, Muller J and Wakeman JA: Brachyury confers cancer stem cell characteristics on colorectal cancer cells. Int J Cancer 130: 328-337, 2012.

35. Palena C, Roselli M, Litzinger MT, Ferroni P, Costarelli L, Spila A, Cavaliere F, Huang B, Fernando RI, Hamilton DH, et al: Overexpression of the EMT driver brachyury in breast carcinomas: Association with poor prognosis. J Natl Cancer Inst 106: pii: dju054, 2014. doi: 10.1093/jnci/dju054. 